\title{
Rapidly Progressing Aseptic Abscesses in a Patient with Ulcerative Colitis
}

\author{
Yoshiharu Yamaguchi, Marie Nakagawa, Shoko Nakagawa, Kazuhiro Nagao, Satoshi Inoue, \\ Tomoya Sugiyama, Shinya Izawa, Yasutaka Hijikata, Masahide Ebi, Yasushi Funaki, \\ Naotaka Ogasawara, Makoto Sasaki and Kunio Kasugai
}

\begin{abstract}
:
Aseptic abscesses (AAs) are extraintestinal manifestations of inflammatory bowel disease (IBD). IBDassociated AAs are rare in Japan. We treated a 45-year-old man with ulcerative colitis (UC)-associated AAs. During remission, multiple progressive abscesses were detected in the spleen; he underwent splenectomy because an infectious disease was suspected. Although his condition improved temporarily after splenectomy, a large liver abscess was noted, and a diagnosis of UC-associated AAs was made. Granulocytapheresis (GCAP) and infliximab (IFX) administration resolved the abscess. This is the first reported case of UC-associated AAs in a Japanese patient treated by splenectomy, GCAP, and IFX.
\end{abstract}

Key words: aseptic abscesses, ulcerative colitis, splenectomy, granulocytapheresis, infliximab

(Intern Med 60: 725-730, 2021)

(DOI: 10.2169/internalmedicine.5733-20)

\section{Introduction}

Aseptic abscesses (AAs) are focal enclosed lesions characterized by the presence of abscesses, failure of antibiotic therapy, and negative blood and aspirate cultures; however, improvement is seen after corticosteroid therapy with or without adjunctive immunosuppressant therapy. AAs are closely related to neutrophilic dermatoses and inflammatory bowel disease (IBD) (1). Although the prevalence of IBD in Japan has increased substantially (2), few cases of IBDassociated AAs have been reported to date (3-5).

We herein report a rare case of IBD-associated AAs in a Japanese patient.

\section{Case Report}

A 45-year-old man was referred to our hospital from an outpatient clinic with a 30-day history of a fever and epigastric pain, weight loss of $8 \mathrm{~kg}$, and skin lesions on both legs. His family history was unremarkable. His bowel habits were unchanged, with 2-3 non-bloody stools per day. He had a 30-year history of pancolonic ulcerative colitis (UC) and ankylosing spondylitis (AS) and was undergoing treatment with mesalazine ( $3.6 \mathrm{~g} /$ day) and adalimumab (ADA) $(40 \mathrm{mg}$ every 2 weeks). The patient's condition was stable with the occasional abdominal pain or slightly bloody diarrhea (partial Mayo score 3).

A physical examination conducted upon his admission was unremarkable, except for hyperthermia $\left(38.8^{\circ} \mathrm{C}\right)$. Laboratory findings (Table 1) showed an erythrocyte sedimentation rate of $57 \mathrm{~mm}$ in the first hour (normal value, $1-7 \mathrm{~mm}$ ); hemoglobin, $8.9 \mathrm{~g} / \mathrm{dL}$ (normal value, 13.9-16 g/dL); white blood cell (WBC) count, $15.3 \times 10^{3} / \mu \mathrm{L}$ (normal value, $5-8 \times$ $10^{3} / \mu \mathrm{L}$ ), with neutrophils at $13,479 / \mu \mathrm{L}$, lymphocytes at $1,056 / \mu \mathrm{L}$, and monocytes at $734 / \mu \mathrm{L}$; platelet count, $448 \times 10^{3} /$ $\mu \mathrm{L}$ (normal value, $138-309 \times 10^{3 /} \mu \mathrm{L}$ ); C-reactive protein (CRP), $11.79 \mathrm{mg} / \mathrm{dL}$ (normal value, $<0.3 \mathrm{mg} / \mathrm{dL}$ ); aspartate aminotransferase, $7 \mathrm{U} / \mathrm{L}$ (normal value, $13 \pm 33 \mathrm{U} / \mathrm{L}$ ); alanine aminotransferase, $6 \mathrm{U} / \mathrm{L}$ (normal 6-30 U/L); total bilirubin, $0.29 \mathrm{mg} / \mathrm{dL}$ (normal value, $0.3-1.2 \mathrm{mg} / \mathrm{dL}$ ); alkaline phosphatase, $205 \mathrm{U} / \mathrm{L}$ (normal value, $115 \pm 359 \mathrm{U} / \mathrm{L}$ ); and gammaglutamyl transferase, $19 \mathrm{U} / \mathrm{L}$ (normal value, 10-47 U/L).

Colonoscopy at the time of admission demonstrated a

Department of Gastroenterology, Aichi Medical University School of Medicine, Japan

Received: June 29, 2020; Accepted: August 9, 2020; Advance Publication by J-STAGE: September 30, 2020

Correspondence to Dr. Yoshiharu Yamaguchi, yamaguchi.yoshiharu.902@mail.aichi-med-u.ac.jp 
Table 1. Results of Blood Examination of the Patient.

\begin{tabular}{lclrlc}
\hline Parameter & Value & Parameter & \multicolumn{1}{c}{ Value } & Parameter & Value \\
\hline ALB & $2.9 \mathrm{~g} / \mathrm{dL}$ & T-Bil & $0.29 \mathrm{mg} / \mathrm{dL}$ & WBC & $15,300 / \mu \mathrm{L}$ \\
UN & $4.5 \mathrm{mg} / \mathrm{dL}$ & Glu & $102 \mathrm{mg} / \mathrm{dL}$ & Neutrophils & $13,479 / \mu \mathrm{L}$ \\
$\mathrm{CRE}$ & $0.61 \mathrm{mg} / \mathrm{dL}$ & $\mathrm{Na}$ & $135 \mathrm{mEq} / \mathrm{L}$ & Lymphocytes & $1,056 / \mu \mathrm{L}$ \\
$\mathrm{AST}$ & $7 \mathrm{U} / \mathrm{L}$ & $\mathrm{K}$ & $3.9 \mathrm{mEq} / \mathrm{L}$ & Monocytes & $734 / \mu \mathrm{L}$ \\
$\mathrm{ALT}$ & $6 \mathrm{U} / \mathrm{L}$ & $\mathrm{Cl}$ & $100 \mathrm{mEq} / \mathrm{L}$ & Eosinocytes & $0.1 \%$ \\
$\mathrm{ALP}$ & $205 \mathrm{U} / \mathrm{L}$ & $\mathrm{Ca}$ & $8.3 \mathrm{mg} / \mathrm{dL}$ & $\mathrm{Hb}$ & $8.9 \mathrm{~g} / \mathrm{dL}$ \\
$\mathrm{LDH}$ & $105 \mathrm{U} / \mathrm{L}$ & $\mathrm{CRP}$ & $11.79 \mathrm{mg} / \mathrm{L}$ & $\mathrm{Ht}$ & $27.5 \%$ \\
$\gamma$-GTP & $19 \mathrm{U} / \mathrm{L}$ & ESR & $57 \mathrm{~mm}$ & Plt & $448 \times 10^{3} / \mu \mathrm{L}$ \\
\hline
\end{tabular}

Alb: albumin, UN: urea nitrogen, CRE: creatinine, AST: aspartate aminotransferase, ALT: alanine aminotransferase, ALP: alkaline phosphatase, LDH: lactate dehydrogenase, $\gamma$-GTP: $\gamma$-glutamyl transpeptidase, T-Bil: total bilirubin, Glu: glucose, Na: sodium, K:potassium, Cl: chloride, Ca: calcium, CRP: C-reactive protein, ESR: erythrocyte sedimentation rate, WBC: white blood cell, Hb: hemoglobin, Ht: hematocrit, Plt: platelet
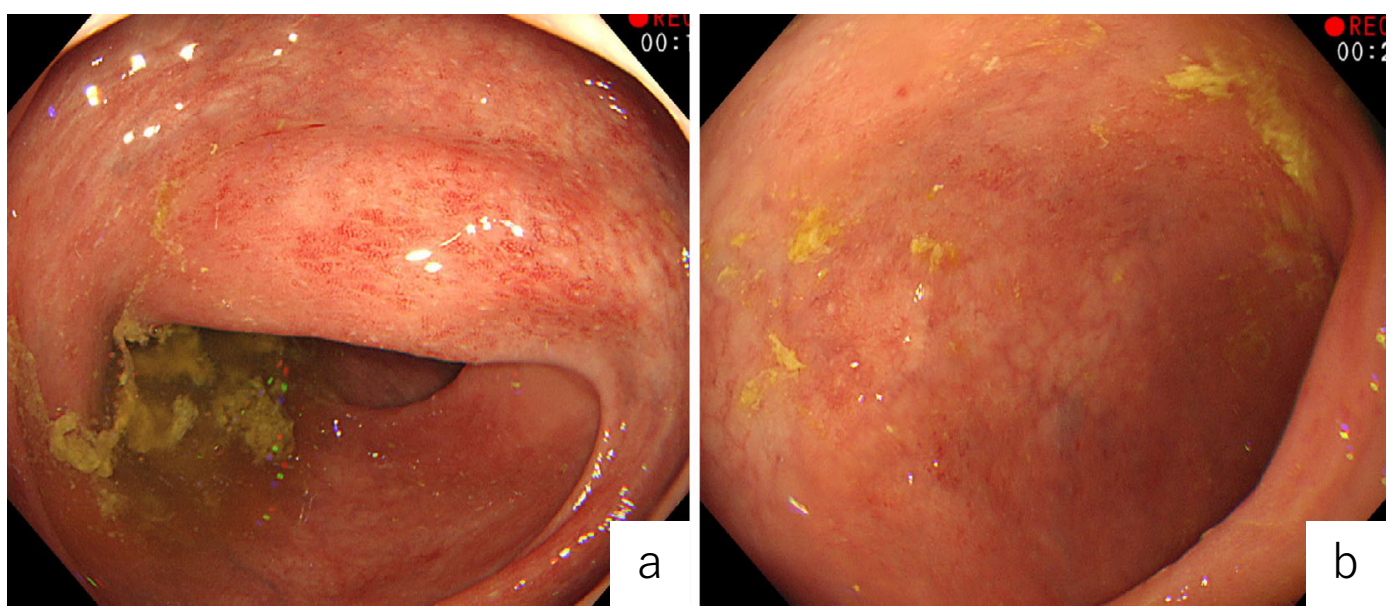

Figure 1. Colonoscopy showed a lack of vascular patterning and friable mucosa (Mayo endoscopic score 1) in the ascending (a) and sigmoid (b) colon.

lack of vascular patterning and friable mucosa in the entire colon (Mayo endoscopic score 1) (Fig. 1a, b). In addition, chest-abdomen-pelvis computed tomography (CT) revealed a hypodense splenic lesion (Fig. 2a), enlarged mesenteric lymph nodes (Fig. 2b), and a lung nodule in the right upper lobe (Fig. 2c). The initial provisional diagnosis was acute bacterial infection or underlying lymphoma, and ADA administration was discontinued; however, mesalazine administration was continued.

Fine-needle aspiration (FNA) of the enlarged mesenteric lymph nodes showed purulent fluid and cultures of the aspiration fluid were negative for the presence of bacteria. A blood culture examination was performed, and the patient was administered intravenous piperacillin/tazobactam (4 g/ $0.5 \mathrm{~g}$, every 8 hours). During hospitalization, he complained of pain in the left side of his abdomen. Despite receiving 18 days of antibiotic therapy since his admission, his pain did not improve, and several cutaneous abscesses and erythematous nodules developed on his legs. However, we continued treatment with broad-spectrum antibiotics because we had not yet obtained any results from the sample cultures or pathological results of FNA.

CT performed 18 days after admission showed an increase in the size and number of splenic lesions (Fig. 2d) and no change in the size of the mesenteric lymph nodes (Fig. 2e); however, the lung nodule had increased in size (Fig. 2f). Blood chemistry showed a WBC count of $23.2 \times$ $10^{3} / \mu \mathrm{L}$ and a CRP level of $17.53 \mathrm{mg} / \mathrm{dL}$.

On the 18th day after admission, splenectomy was performed. The resected spleen showed multiple abscesses (Fig. 3a). A histological examination revealed necrotic tissue containing polymorphonuclear leucocytes surrounded by palisading histiocytes (Fig. 3b, c). Cultures of samples obtained from the blood, lymph nodes, spleen, and skin were negative for aerobic and anaerobic bacteria, fungi, and mycobacteria. Three weeks after splenectomy, he was discharged as the laboratory findings showed a dramatic improvement in the inflammatory parameters (WBC count: 7.2 $\times 10^{3} / \mu \mathrm{L}$; CRP: $1.83 \mathrm{mg} / \mathrm{dL}$ ) and the dermatologic lesions had disappeared.

One month after his discharge, he was admitted to our hospital with a 3-day history of a fever, abdominal pain, and 

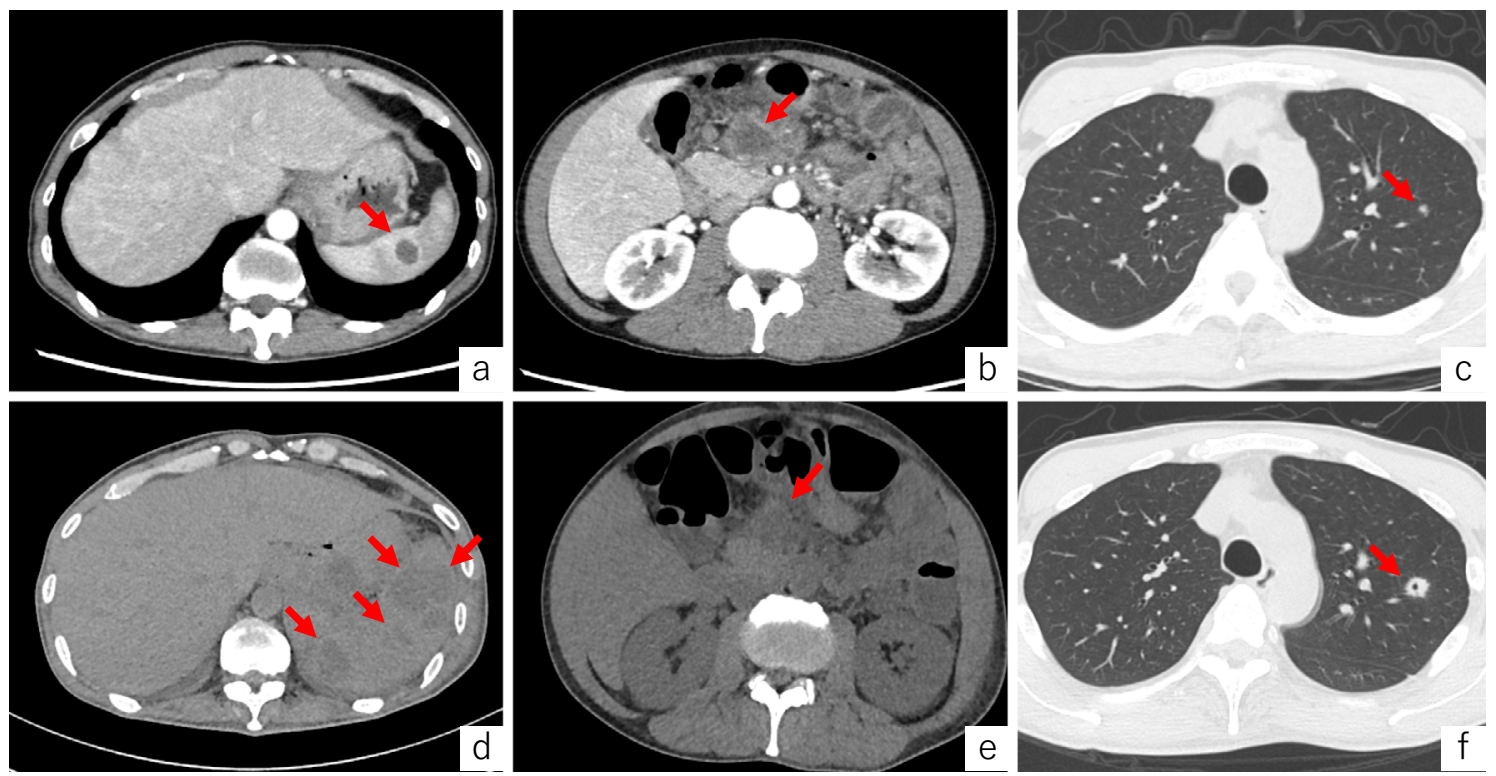

Figure 2. Axial contrast-enhanced computed tomography shows splenic hypodense abscess (arrow) (a), enlarged mesenteric lymph nodes (arrow) (b), and a lung nodule in the right upper lobe (arrow) (c). For 10 consecutive days, the splenic lesions increased in size and number (arrows) (d); however, the mesenteric lymph nodes showed no changes (arrow) (e), and the lung nodule increased in size and formed a cavity (arrow) (f).
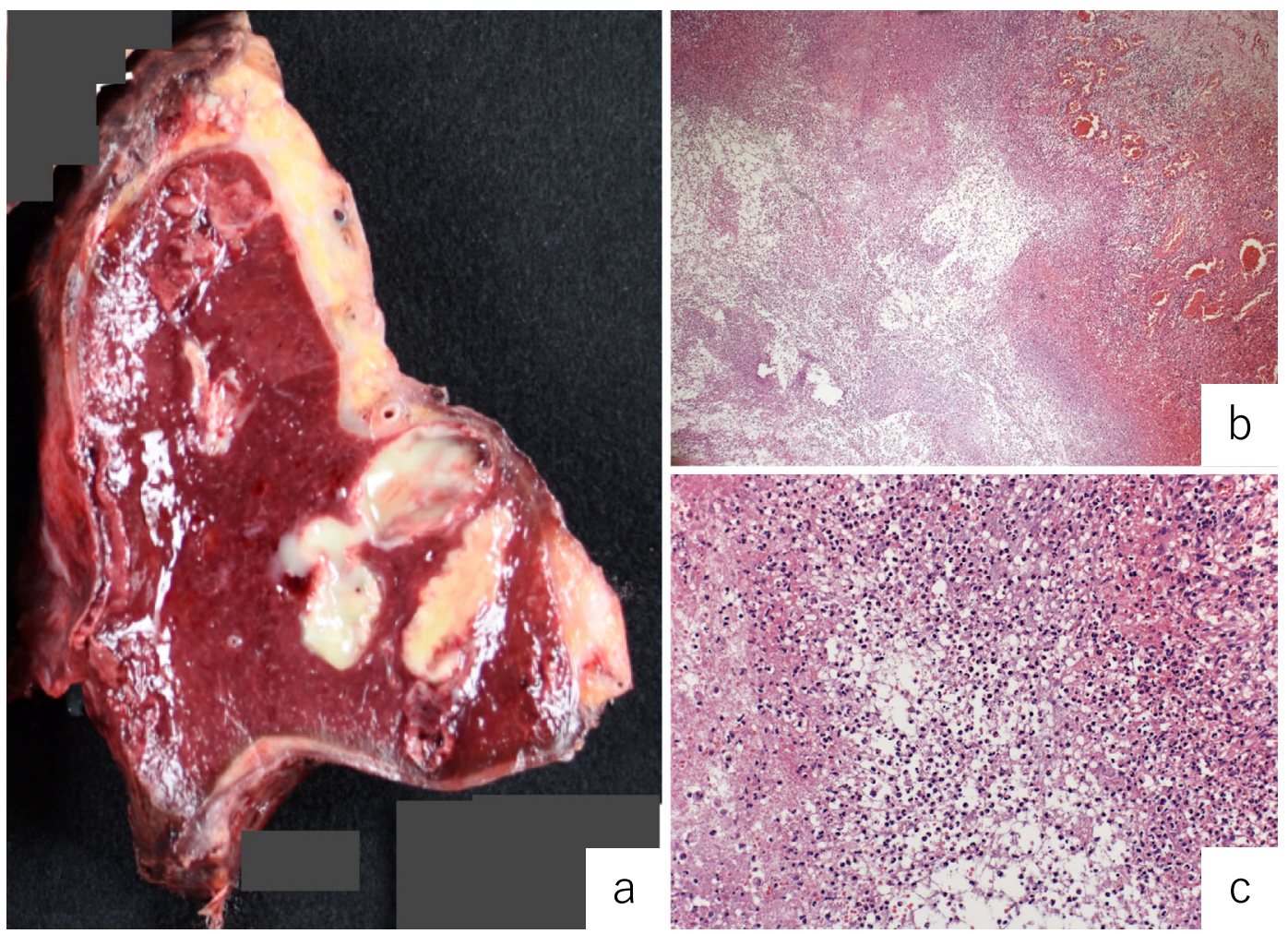

Figure 3. Macroscopic examinations of the split surface of the spleen show multiple yellow nodular lesions (a). A histological examination show necrotic tissue (b) with polymorphonuclear leucocytes surrounded by palisading histiocytes (c).

the reappearance of skin lesions on both legs. However, he reported no marked changes in his bowel habits because of the administration of mesalazine. Abdominal and pelvic CT showed a solitary cystic liver mass of $8.1 \mathrm{~cm}$ (Fig. 4a).
Blood chemistry revealed a WBC count of $15.7 \times 10^{3} / \mu \mathrm{L}$ and a CRP level of $13.73 \mathrm{mg} / \mathrm{dL}$. The aspirated fluid of the liver abscess was cultured for bacteria and demonstrated that the liver abscess was sterile. The patient was diagnosed with 


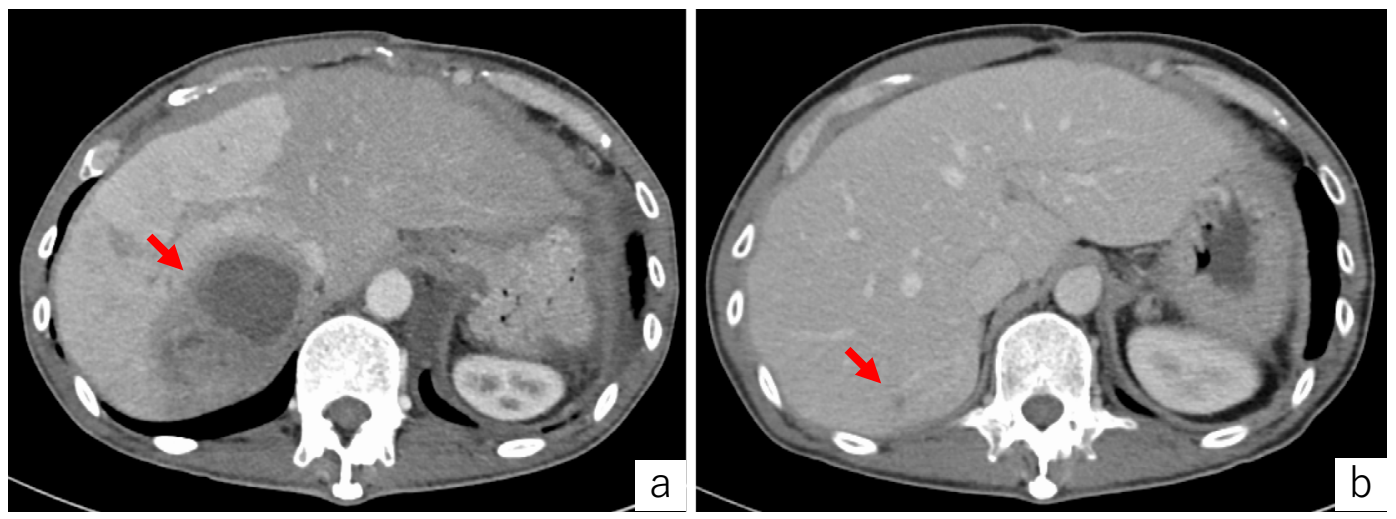

Figure 4. Contrast-enhanced computed tomography shows a large, hypodense lesion with irregular margins in the liver, and the right hepatic lobe shows an increased density (arrow) (a). After three months, the hypodense lesion decreased in size (arrow) (b).

Table 2. Clinical Findings and Treatment Data of Patients with the Inflammatory Bowel Disease-associated Aseptic Abscesses from the Literature Database and Our Reported Case.

\begin{tabular}{|c|c|}
\hline Reference/Number of patients & Total. 1994 to $2020[1,6-8$, our case $] / 43$ \\
\hline Age/sex & Mean 29.7, range10-80/27F\&16M \\
\hline IBD phenotype (CD/UC/IC) & $29 \mathrm{CD} / 13 \mathrm{UC} / 1 \mathrm{IC}$ \\
\hline $\begin{array}{l}\text { Age of IBD diagnosis/temporal relation to } \\
\text { diagnosis of AA }\end{array}$ & $\begin{array}{l}\text { Average age of IBD onset }=25.4(\text { range } 10-72,3 \text { unknown }) / \text { before }(n=23) \text {, } \\
\text { concomitant }(n=11), \text { after }(n=9)\end{array}$ \\
\hline IBD flare during AA & $\operatorname{Yes}(\mathrm{n}=20)$, no $(\mathrm{n}=23)$ \\
\hline Symptoms & Fever $(n=36)$, abdominal pain $(n=30)$, weight loss $(n=16)$, diarrhea $(n=14)$ \\
\hline Location of AA & $\begin{array}{l}\text { Spleen }(n=21) \text {, liver }(n=9) \text {, lymph nodes }(n=13) \text {, muscles }(n=2) \text {, sternum }(n=2) \text {, } \\
\text { epidural }(n=2) \text {, and eyelids, pancreas, kidney, pharynx, scalp, face, inner } \\
\text { canthus, submaxilla, chest, forearm, nasal septum }(n=1 \text {, respectively })\end{array}$ \\
\hline Other EIM of IBD & $\begin{array}{l}\text { Arthritis }(n=10) \text {, myalgia }(n=5) \text {, neutrophilic dermatosis }(n=2), P G(n=4) \text {, } \\
\text { sweet's syndrome }(n=2) \text {, aphthous ulcer }(n=8) \text {, and abscesses of his lower legs } \\
\text { with caseating granulomas, panniculitis, polyneuropathy }(n=1 \text {, respectively) }\end{array}$ \\
\hline Antibiotic treatment & Yes $(n=39), \operatorname{no}(n=4)$ \\
\hline Corticosteroids & Yes $(n=39), \operatorname{no}(n=4)$ \\
\hline Additional immunotherapy & $\begin{array}{l}\text { Total }(n=18), \text { cyclophosphamide }(n=3) \text {, azathioprine }(n=12), \\
\text { methotrexate }(n=1), \operatorname{GCAP}(n=1) \text {, adalimumab }(n=1) \text {, infliximab }(n=5)\end{array}$ \\
\hline Surgical procedures & $\begin{array}{c}\text { Splenectomy }(\mathrm{n}=17), \text { incision and drainage }(\mathrm{n}=5) \text {, laparoscopic biopsy of } \\
\text { mesenteric lymph nodes }(\mathrm{n}=1)\end{array}$ \\
\hline Maintenance therapy after diagnosis of AA & $\begin{array}{l}\text { Information not provided }(\mathrm{n}=30) \text {, prednisone }(\mathrm{n}=4) \text {, azathioprine }(\mathrm{n}=2) \text {, } \\
\text { sulfasalazine }(\mathrm{n}=1), 5-\operatorname{ASA}(\mathrm{n}=3) \text {, adalimumab }(\mathrm{n}=1) \text {, infliximab }(\mathrm{n}=4)\end{array}$ \\
\hline Number of relapses & Mean 1.02, range $0-5(n=34)$ \\
\hline
\end{tabular}

AA: aseptic abscess, CD: Crohn's disease, EIM: extraintestinal manifestation, F: female, GCAP: granulocytapheresis, IC: indeterminate colitis, IBD: inflammatory bowel disease, M: male, PG: pyoderma gangrenosum, UC: ulcerative colitis

UC-associated AAs and pyoderma gangrenosum (PG).

Granulocytapheresis (GCAP) (10 passages) and the intravenous administration of infliximab (IFX) $(5 \mathrm{mg} / \mathrm{kg}$, every 8 weeks) were started. As the patient became afebrile, the cutaneous lesions and hepatic abscess improved (Fig. 4b). The mesenteric lymph nodes and lung nodule maintained their sizes before the treatment. However, seven months after IFX administration, the patient was followed up as an outpatient and found to be free of any clinical symptoms.

\section{Discussion}

To our knowledge, only 43 patients with IBD-associated AAs have been documented and identified using MEDLINE.
The features of IBD-associated AAs identified from these reported cases are summarized in Table 2 (1, 6-8). Our patient is the third Japanese patient reported to have IBD with AAs and the first Japanese patient to have IBD-associated AAs in the spleen, mesenteric lymph nodes, lung, and liver who underwent treatment with splenectomy, GCAP, and IFX.

AAs is a neutrophilic disease of unknown cause that forms chronic recurrent abscesses, frequently located in the spleen, abdominal lymph nodes, and liver. Histopathological examinations have shown predominant infiltration of neutrophils around the granulomatous tissue and sterile abscesses. The systemic administration of steroids is the most common induction treatment, and the efficacy of anti-tumor necrosis 
factor- $\alpha$ (TNF- $\alpha)$ antibody in addition to steroid pulse therapy and immunosuppressants has been recently reported in some refractory cases (Table 2) (1, 6-8). An AA diagnosis is a diagnosis of exclusion, and there is currently no established diagnostic method. However, Andre et al. reported the following diagnostic clues for AAs: (a) deep abscesses with neutrophilic features; (b) negative serologic tests for bacteria and fungi and cultures of blood and aspirate; (c) when administered, failure of broad-spectrum antibiotic therapy, including antituberculosis therapy; and (d) rapid clinical improvement on corticosteroid therapy with or without additional immunosuppressant therapy and subsequent radiologic evidence of abscess resolution (9). Our case presented with these features of AAs. If a patient has a background of diseases with immune abnormalities, the diagnosis should be reached as soon as possible using evidence to reject the possibility of infection and prove the pathology.

In our case, multiple splenic abscesses were initially suspected to have been caused by bacterial infection. For this reason, the patient underwent splenectomy, and the abscesses were found to be sterile. Although there were many possibilities of infections causing similar life-threating abscesses (10), we could not initially exclude all infections in this case. Therefore, we chose to perform splenectomy to arrive at an exact diagnosis.

Because of the considerable overlap with other infections based on the appearance on imaging modalities, such as ultrasound, CT, and MRI, and the small number of AAs, AAs are extremely difficult to distinguish. Abscess formation is essentially a persistent and intense inflammatory irritation, so it is unlikely that differences in abscess formation found on imaging studies due to variable causes of irritation can be noted.

To prove that the abscesses are sterile, an invasive examination, such as laparotomy, is required. Furthermore, a temporary improvement in systemic symptoms after splenectomy can be attributed to a decrease in the systemic immune response, owing to the removal of the largest accumulation of lymphoid tissue, which controls immunity, in the body (11).

The present patient refused the administration of steroids, and eventually, the symptoms improved after splenectomy with GCAP and IFX.

The event in the present patient occurred during the use of ADA; however, we did not use ADA again because ADA's secondary loss of response could not be ruled out. Furthermore, we did not use steroids due to the lack of clarity regarding the accumulation of steroids during long-term UC treatment and the patient's strong refusal of steroids, which required long-term administration after hospital admission. Therefore, IFX treatment was chosen in this case. AA remission can be induced without using steroids and sustained with GCAP and IFX. GCAP is reported to be useful for the treatment of IBD and PG $(12,13)$. Indeed, Kato et al. described a Japanese patient with AAs and Crohn's disease $(\mathrm{CD})$ who showed improvement following treatment with GCAP (5). The advantages of GCAP are that it rarely causes adverse events and is considered to be as useful as corticosteroids for induction therapy of AAs.

The precise mechanism underlying the action of GCAP has not been clearly described. However, GCAP selectively adsorbs and removes activated monocytes and MAC-1expressing neutrophils that infiltrate the inflamed regions. In addition, GCAP reduces the circulating levels of inflammatory cytokines, such as TNF- $\alpha$, interleukin (IL)- $1 \beta$, IL-6, and IL- $8(14,15)$. Therefore, GCAP is effective for treating AAs as a systemic disease.

Andre et al. reported that $60 \%$ of AAs relapsed at least once in a 7-year follow-up period (9). Our patient required careful follow-up when he was on remission maintenance therapy. When a patient experiences a relapse, novel antibody formulations, such as vedolizumab, which is effective for $\mathrm{PG}$ associated with $\mathrm{CD}$, and ustekinumab, which has been reported to improve inflammatory cutaneous lesions, are expected to be effective $(16,17)$.

Possible mechanisms underlying the pathogenesis of AAs associated with UC have been discussed. The pathology of UC, AAs, PG, and spondylitis is considered to be related to aseptic neutrophil infiltration. Pathological conditions based on neutrophil dysfunction were considered to have resulted in the condition of our patient. The patient had no family history of a similar disease; however, he did not wish to undergo genetic testing, such as DNA typing, to determine if he had any genetic mutations. Therefore, these tests were not performed. However, human leukocyte antigen (HLA)B27 is positive in $25-78 \%$ of patients with concomitant IBD and AS. In addition, other mutations, such as those in the PSTPIP1 gene, which cause pyogenic arthritis, pyoderma gangrenosum, and acne syndrome, may also be present $(18,19)$.

Extraintestinal manifestations of IBD correlate with the IBD activity. In contrast, AS and PG associated with IBD have no correlation with the IBD activity $(18,20)$. In our case, AS and PG did not correlate with the IBD activity, and AA did not correlate with the UC activity. However, the AA and PG activity were correlated. We must be careful when managing cases with AAs without IBD relapse, as AAs may not be related to the intestinal activity. Further research will be needed to determine the relationship between AAs and IBD.

Risk factors for AAs may include a high likelihood of skin conditions. However, the number of cases has been small (43 cases, including our case), so the risk factors are not clear. Therefore, it will be necessary to clarify these risk factors by accumulating more cases in the future.

Given the present findings, even when patients are in remission in chronic IBD, gastroenterologists must pay close attention to AAs and provide prompt treatment.

The authors state that they have no Conflict of Interest (COI). 


\section{References}

1. Bollegala N, Khan R, Scaffidi MA, et al. Aseptic abscesses and inflammatory bowel disease: two cases and review of literature. Can J Gastroenterol Hepatol 2017: 5124354, 2017.

2. Murakami Y, Nishiwaki Y, Oba MS, et al. Estimated prevalence of ulcerative colitis and Crohn's disease in Japan in 2014: an analysis of a nationwide survey. J Gastroenterol 54: 1070-1077, 2019.

3. Maeshima K, Ishii K, Inoue M, Himeno K, Seike M. Behçet's disease complicated by multiple aseptic abscesses of the liver and spleen. World J Gastroenterol 19: 3165-3168, 2013.

4. Ito T, Sato N, Yamazaki H, Koike T, Emura I, Saeki T. A case of aseptic abscesses syndrome treated with corticosteroids and TNFalpha blockade. Mod Rheumatol 23: 195-199, 2013.

5. Kato S, Hosomi E, Amano F, et al. The efficacy of intensive granulocyte and monocyte adsorption apheresis in a patient with Crohn's disease complicated by extensive subcutaneous aseptic neutrophilic abscesses. J Crohns Colitis 6: 787-791, 2012.

6. Yang Y, Chen D. Treatment of aseptic liver abscess due to Crohn's disease using infliximab. Clin Gastroenterol Hepatol 15: A27-A28, 2017.

7. Herskovitz I, Maderal AD, Alonso-Llamazares J. Caseating granulomas manifesting as aseptic abscesses in the setting of ulcerative colitis. Int J Dermatol 57: 475-476, 2018.

8. Bavaro DF, Ingravallo G, Signorile F, et al. Splenic abscesses as a first manifestation of Crohn's disease: a case report. BMC Gastroenterol 19: 144, 2019.

9. Andre MF, Piette JC, Kemeny JL, et al. Aseptic abscesses: a study of 30 patients with or without inflammatory bowel disease and review of the literature. Medicine (Baltimore) 86: 145-161, 2007.

10. Kamaya A, Weinstein S, Desser TS. Multiple lesions of the spleen: differential diagnosis of cystic and solid lesions. Semin Ultrasound CT MR 27: 389-403, 2006.

11. Hansen K, Singer DB. Asplenic-hyposplenic overwhelming sepsis: postsplenectomy sepsis revisited. Pediatr Dev Pathol 4: 105-121, 2001.
12. Matsuda K, Ohno K, Okada Y, et al. Adsorptive granulocyte and monocyte apheresis is effective in ulcerative colitis patients both with and without concomitant prednisolone. Inflamm Intest Dis 5: 36-41, 2020.

13. Russo I, Miotto S, Colpo A, et al. Successful treatment of pyoderma gangrenosum with granulocyte and monocyte adsorption apheresis. Int Wound J 14: 282-284, 2017.

14. Hanai H, Takeda Y, Eberhardson M, et al. The mode of actions of the Adacolumn therapeutic leucocytapheresis in patients with inflammatory bowel disease: a concise review. Clin Exp Immunol 163: 50-58, 2011.

15. Cuadrado E. Granulocyte/monocyte apheresis as immunotherapic tool: cellular adsorption and immune modulation. Autoimmun Rev 8: 292-296, 2009.

16. Vernero M, Ribaldone DG, Cariti C, et al. Dual-targeted therapy with apremilast and vedolizumab in pyoderma gangrenosum associated with Crohn's disease. J Dermatol 47: e216-e217, 2020.

17. Phillips FM, Verstockt B, Sebastian S, et al. Inflammatory cutaneous lesions in inflammatory bowel disease treated with Vedolizumab or Ustekinumab: an ECCO CONFER multicentre case series. J Crohns Colitis 14: 1488-1493, 2020.

18. Ott C, Scholmerich J. Extraintestinal manifestations and complications in IBD. Nat Rev Gastroenterol Hepatol 10: 585-595, 2013.

19. Martinez-Rios $C$, Jariwala MP, Highmore $K$, et al. Imaging findings of sterile pyogenic arthritis, pyoderma gangrenosum and acne (PAPA) syndrome: differential diagnosis and review of the literature. Pediatr Radiol 49: 23-36, 2019.

20. Marzano AV, Borghi A, Stadnicki A, Crosti C, Cugno M. Cutaneous manifestations in patients with inflammatory bowel diseases: pathophysiology, clinical features, and therapy. Inflamm Bowel Dis 20: 213-227, 2014.

The Internal Medicine is an Open Access journal distributed under the Creative Commons Attribution-NonCommercial-NoDerivatives 4.0 International License. To view the details of this license, please visit (https://creativecommons.org/licenses/ by-nc-nd/4.0/).

(C) 2021 The Japanese Society of Internal Medicine Intern Med 60: 725-730, 2021 